\title{
Ultrasound Contribution to the Diagnosis of Emphysematous Cystitis
}

\author{
Kofi-Mensa Savi de Tové1, Madina Napon², Zakari Nikiema3 ${ }^{3}$ Patricia Yèkpè̀, \\ Olivier Biaou4, Vicentia Boco ${ }^{4}$ \\ ${ }^{1}$ Medical Imaging Department, Faculty of Medicine, University of Parakou, Republic of Benin \\ ${ }^{2}$ Medical Imaging Unit, Charles de Gaulle University Teaching Hospital, Ouagadougou, Burkina-Faso \\ ${ }^{3}$ Medical Imaging Unit, Sourô Sanou University Teaching Hospital, Bobo-Dioulasso, Burkina-Faso \\ ${ }^{4}$ Medical Imaging Department, Faculty of Health Sciences, University of Abomey-Calavi, Cotonou, \\ Republic of Benin \\ Email: savitoveto@yahoo.fr
}

Received 28 August 2015; accepted 25 September 2015; published 28 September 2015

Copyright (C) 2015 by authors and Scientific Research Publishing Inc.

This work is licensed under the Creative Commons Attribution International License (CC BY).

http://creativecommons.org/licenses/by/4.0/

(c) (i) Open Access

\section{Abstract}

Emphysematous cystitis is a severe urinary tract infection which threatens patients' life. Thus, it requires early diagnosis and computed tomography (CT) is the reference medical exam used to address it. However, ultrasound, which is a non-ionizing and very accessible technique, may also contribute to diagnosis. The aim of this study was to illustrate the contribution of ultrasound to the diagnosis of emphysematous cystitis. We report three cases of emphysematous cystitis diagnosed by ultrasound. In all the cases, ultrasound identified basic signs showing presence of gas, including a hyperechoic cystic parietal thickening, repeat echoes and dirty shadow cones of intraluminal focus. Diagnostic confirmation was made in two cases through X-ray and in one case by means of computed tomography. Through simple basic signs, ultrasound directs most usefully towards an emphysematous urinary tract infection. CT enables a more accurate diagnostic work-up of lesions and differential diagnosis with vesico-digestive fistula.

\section{Keywords}

Emphysematous Cystitis, Ultrasound, Computed Tomography, X-Ray

\section{Introduction}

Emphysematous cystitis is an acute urinary infection which threatens patients' life [1]. It is an infrequent infection characterized by gas presence in bladder lumen and/or wall. Most of times it is a urinary infection outcome 
attended with delay. Diabetes and urinary tract obstructions are risk factors [2]. The bacteria that are more commonly identified are Escherichia coli and Klebsiella pneumoniae. The clinical signs of emphysematous cystitis are variable; they range from uncomplicated pollakiuria to abdominal pain with hematuria; sometimes, disorders of consciousness may be indicative factors. The clinical signs suggesting emphysematous cystitis appear lately and are not specific enough [1]. Treatment is based on triple antibiotic therapy adapted to antimicrobial susceptibility testing associated with bladder drainage, and in diabetic patients to glycemic control. This disease severity is mainly associated with late diagnosis and treatment. Medical imaging contributes to early diagnosis by identifying air and specifying location on the urinary tract. Several imaging tools are used for their diagnosis and among them CT has emerged as a reference exam [3]-[5]. Nevertheless, due to the poor specificity of clinical signs, computed tomography is not always the first medical imaging exam used. Besides, this technique is not accessible enough in developing countries, including Benin. Ultrasound, a non-ionizing and more accessible technique, can significantly contribute to diagnosis [6] [7]. In the literature, it is not commonly used as a diagnostic tool [1] [8]. However, according to many authors, it would have sensitivity similar to the one of cystoscopy which is another tool for emphysematous cystitis diagnosis. The study of three cases of emphysematous cystitis enabled the authors to emphasize ultrasound contribution to the diagnosis of those horrific infections.

\section{Observations}

\subsection{Observation 1}

Mr. X, a 58-year-old man, had been admitted for vesico-prostatic ultrasound concerning a pollakiuria he was suffering from for about one week. The patient is suffering from type 2 diabetes diagnosed for about ten years and is put on synthetic oral diabetes drugs.

The clinical exam noted $38^{\circ} \mathrm{C}$ hyperthermia and pain during hypogastrium palpation which produced tympanic resonance on percussion. The biological test detected a $2.5 \mathrm{~g} / \mathrm{dl}$ hyperglycemia without glycosuria or acetonuria, a hyperleukocytosis of $10,000 \mathrm{~mm}^{3}$ with $70 \%$ of polymorpho-nuclear leukocytes and C-reactive protein of $24 \mathrm{mg} / \mathrm{dl}$. Kidney function test was normal.

Ultrasound identified the existence of a stage 2 muscle bladder and hypertrophy of prostate median lobe (Figure 1(a) and Figure 1(b)). The examination of anterior bladder wall helped note an irregularity of the latter with suspicion of subserous extraparietal hyperechogenicity, thus suggesting diagnosis of emphysematous cystitis (Figure 1(c)). In addition, a bilateral hydronephrosis with suspicion of left emphysematous pyelonephritis (Figure 1(d)) was also identified. Computed tomography enabled to confirm the diagnosis of emphysematous cystitis complicated by a subserosal rupture associated with urethritis (Figure 1(e)) and left emphysematous pyelitis (Figure 1(f)). The outcome improved after bladder ultrasound and triple antibiotic therapy. Urine drainage by bladder catheterization was put in place. Triple antibiotic therapy and diabetes balance were applied through insulin therapy. Under this treatment, outcome is positive with gradual improvement of patient general condition characterized by a normal glycemia three (03) days later. An adenomectomy was performed 45 days later with uncomplicated surgical outcomes.

\subsection{Observation 2}

Mr. X, 69 years of age, hospitalized for urinary tract retention, is referred for performance of vesico-prostatic ultrasound. One month before, he benefitted from surgical pulpectomy for prostatic adenocarcinoma. Clinically, there were infectious syndrome, obnubilation and mere dimness, conjunctival icterus and tympanic bloated abdomen.

The biological tests confirmed the infectious injury by detecting a $25.1 \mathrm{G} / \mathrm{L}$ hyperleukocytosis with $85 \%$ of polymorphonuclear leukocytes and CRP estimated at $96 \mathrm{mg} / \mathrm{ml}$. Moreover, transaminases (AST $=186.12 \mathrm{U} / \mathrm{L}$; $\mathrm{ALT}=127.28 \mathrm{U} / \mathrm{L})$ increased. Hyperuricemia $(2.89 \mathrm{~g} / \mathrm{L})$ and hypercreatininemia $(51.6 \mathrm{mg} / \mathrm{L})$ also disturbed kidney function. Glycemia was normal $(1.04 \mathrm{~g} / \mathrm{L})$. Klebsiella pneumoniae had been isolated in urine.

Ultrasound (Figure 2(a) and Figure 2(b)) permitted to suggest emphysematous cystitis. This diagnosis had been confirmed by X-rays without abdominal preparation (Figure 2(c) and Figure 2(d)). Furthermore, during ultrasound it was noted gallbladder parietal thickening which may cause acalculous cholecystitis.

Once diagnosis of emphysematous cystitis complicated by acalculous cholecystitis was adopted, a triple antibiotic therapy adapted to antimicrobial susceptibility testing and hydro-electrolytic resuscitation was initiated. Bladder drainage with transurethral catheter was also set up. It was noted that patient outcome improved; 


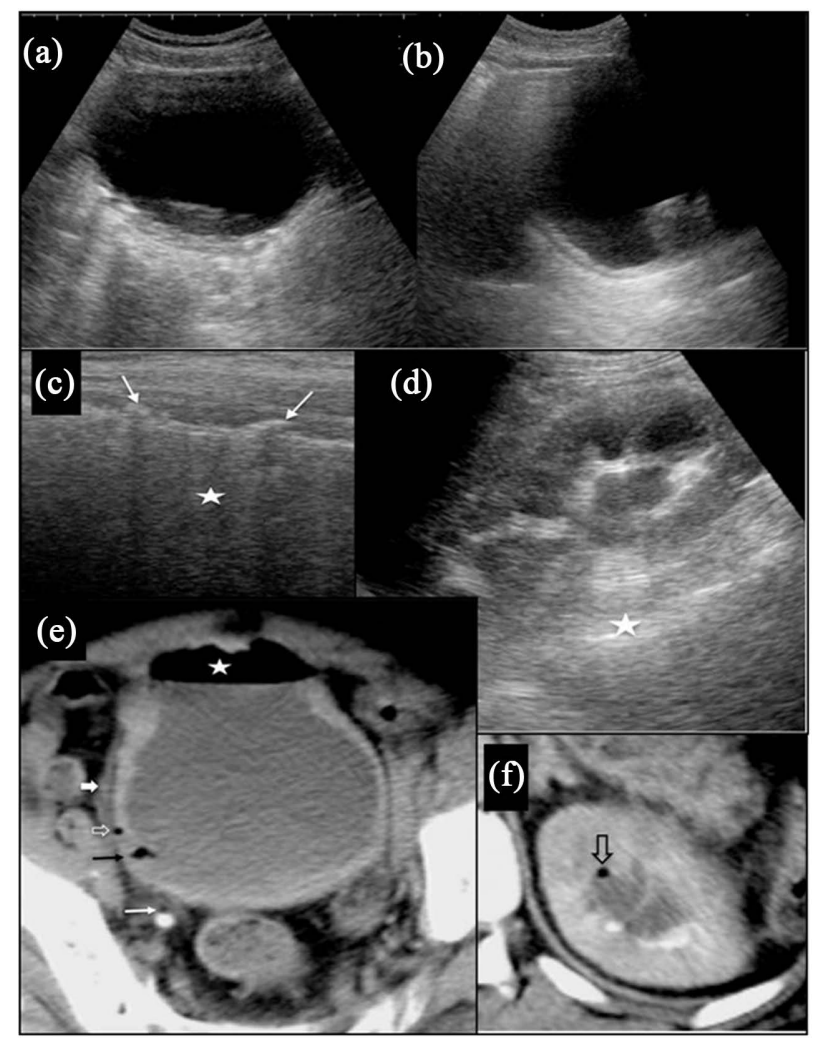

Figure 1. Suprapubic ultrasound: cross-sectional (a) and cross-sectional (b). It showed an hyperechoic area of the anterior bladder wall (vertical white arrows) as indicator of intraparietal air, reverberation echoes and dirty shadow cones associated with intravesical air (5-pointed star). Existence of a posterior parietal and bladder thickening with column, characterized by aerial hyperechogenicities which seem to be tightly held between the columns (horizontal white arrows) and causing shadow cones (arrowheads). There is a hypertrophic median lobe pushing into the posterior lower part of the bladder face located down the back of the ureteric fold (4-pointed star). Ultrasound image of the anterior bladder wall with a superficial probe (c); the hyperechoic aspect of the wall with reverberation echoes backwards is identified (5-pointed star). The wall is irregular and characterized by the presence of more superficial gaseous bubbles (arrows). Longitudinal ultrasound scans of left kidneys (d); there is a pyelocaliceal dilatation with presence of repeat echoes suggesting presence of gas (5-pointed Star). Axial tomographic scan of the bladder's iodinated contrast agent (e) which showed an irregular parietal bladder thickening with presence of intracystic (5-pointed Star) and intraparietal (black arrow) air. We note peripheral air bubble (hollow arrow) implying fluid accumulation in favor of subserosal bladder rupture (shaded arrow). Down the back of bladder, an air bubble is noted in the left ureter (white arrow). CT axial scanof the left kidney (f). Hydronephrosis and left pyelic air bubble (hollow arrow).

clinical signs were completely resolved within one week. As a result, patient was discharged. Bladder catheter was removed later.

\subsection{Observation 3}

It was a 63-year-old female patient referred for ultrasound exploration of urinary retention. She was suffering from type 2 diabetes and was put on synthetic oral diabetes drugs. Physical examination resulted in a slight and short $37.9^{\circ} \mathrm{C}$ fever and a full bladder with tympanic resonance on percussion. The biological tests indicated that CRP rose to $36 \mathrm{mg} / \mathrm{ml}$ with $8000 \mathrm{~GB} / \mathrm{mm}^{3}$ of large amount of polymorphonuclear leukocytes (60\%). The presence of hyperglycemia at $4.6 \mathrm{~g} / \mathrm{dl}$ as well as of acetonuria and glycosuria was noted. Kidney function proved to be normal. Emphysematous cystitis was diagnosed during ultrasound (Figure 3(a)). It had been confirmed during unprepared abdominal examination (Figure 3(b)). Triple antibiotic therapy was performed during 10 days. Insulin therapy permitted to rebalance patient. Bladder drainage with urinary catheter was put in place. Outcome improved under this treatment with normalization of biological parameters and patient discharge after ten (10) days of hospitalization. 


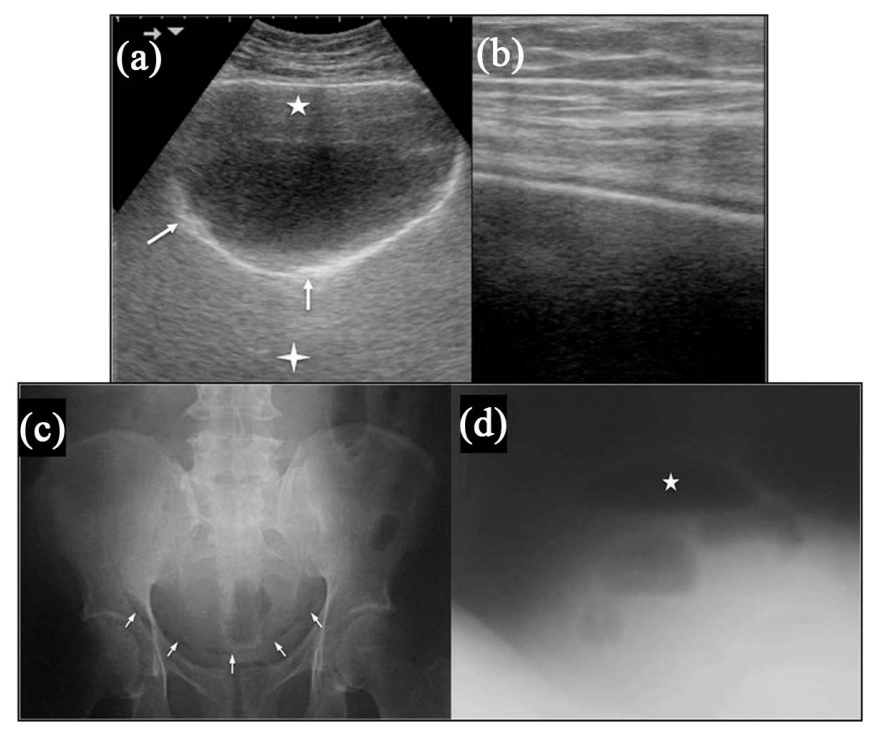

Figure 2. Cross-sectional suprapubic ultrasound scan with a convex probe (a) and a superficial linear probe (b). There is a hyperechoic bladder wall of irregular aspect in the posterior part (white arrows). There is also presence of repeat echoes down the back of anterior bladder wall suggesting existence of intraluminal air (5-pointed star). There is a diffraction of echoes which impedes the visualization of retrovesical pelvic structures (4-pointed star). Exploration with linear probe (b) provides a better analysis of the anterior bladder wall with regular hyperechoic border and diffraction of echoes down the back of the latter. Unprepared negative of pelvis in frontal (c) and profile spine positions (d). Presence of a concave arcuate linear hyperlucency at the top of the iliopelvic branches. This is suggestive of cystic intraparietal air (white arrows) and hypogastric fluid-air level under the anterior abdominal wall associated with presence of intracystic air (5-pointed Star).

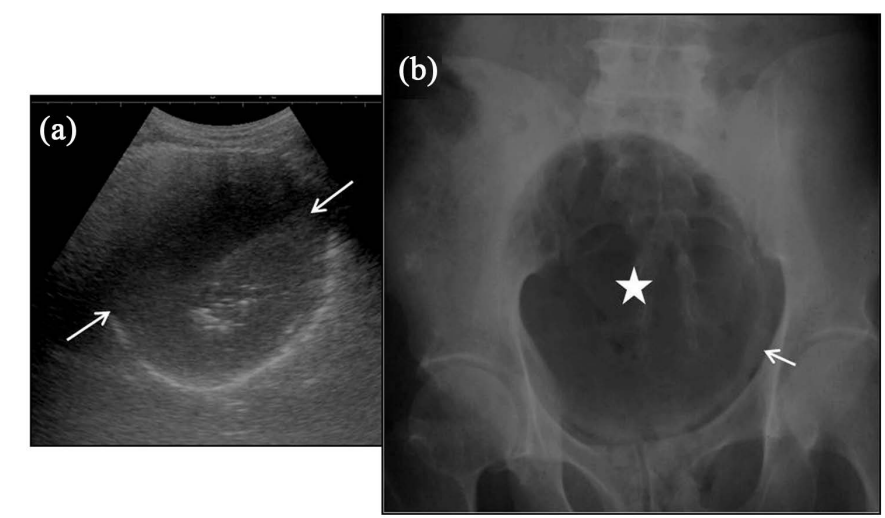

Figure 3. Cross-sectional suprapubic ultrasound with a convex probe (a) showing an irregular hyperechoic bladder wall (white arrows). Retrovesical pelvic structures are not visualized (4-pointed Star). Bladder content is heterogeneous with presence of liquid level suggestive of pyuria (arrow). Unprepared radiography in supine position with vertical radius (b) identifying distended bladder with air content (5-pointed star) with spontaneous visualization of the bladder wall (white arrow).

\section{Discussion}

Emphysematous cystitis is a necrotizing urinary infection characterized by gas presence in the urinary tract. That air presence in the urinary tract had been described for the first time in 1671 in a male subject with pneumaturia. The first emphysematous infection of the bladder had been discovered by the end of the 1800s by Eisenlohr during an autopsy. Bailey named that disease “emphysematous cystitis” in 1961 [1].

The carbon dioxide found in the bladder lumen and/or walls during emphysematous cystitis (EC) is the outcome of carbohydrates' bacterial fermentation; it is an indicator of anaerobic bacterial breathing. The bacteria incriminated are aerobic or anaerobic bacteria such as Escherichia coli, Klebsiella pneumoniae, Entero-bacteraerogenes and Proteus mirabilis [1] [3] [8]. 
Most of times, as in our three cases, patients aged around sixty years suffered from those infections. The main risk factors are diabetes mellitus and obstructive uropathy identified in two of our patients [1] [2] [8] [9].

The clinical symptoms of those infections are not specific enough and patients are often wrongly diagnosed for simple cystitis. Classic pneumaturia is found out in few cases [1]. The existence of obstruction on the lower tract may generate full bladder with tympanic resonance on percussion. This lack of specificity of clinical signs explains the importance of medical imaging in emphysematous cystitis diagnosis. Imaging will, through different techniques, visualize the air produced by that bacterial breathing. X-ray shows intraparietal air in the form of air content bordering on the bladder and outlining its shape. The intraparietal air is represented by a hypogastric hydro-aeric level. Its sensitivity depends on the amount of air. At most it is characterized by a gaseous distension of the bladder. Unprepared radiography (X-ray) has no more much importance since the introduction of computed tomography as reference exam for diagnosis and monitoring of emphysematous cystitis. It easily points out the presence of intracystic and intraparietal air. It is the most sensitive exam; moreover it has the advantage of helping eliminate vesico-vaginal fistula which is the main differential diagnosis of emphysematous cystitis [5].

Unfortunately, this technique is not frequently used as a first-choice one in developing countries, including Benin where its availability is limited. Ultrasound is descrambling exam performed as a first-choice operation in the presence of poorly specific symptoms as in the case of emphysematous cystitis. In this series, it allowed emphysematous cystitis diagnosis in our three patients. The initial diagnosis made by ultrasound is not frequently described in the literature. According to Grupper, ultrasound scan would be a contributive factor in only $46 \%$ of the cases [8]. It is a useful technique although its sensitivity is worse than the one of reference technique i.e. CT. It may be an efficient diagnostic tool when CT is not available. A simple basic symptomatology but sometimes difficult to identify helps diagnose emphysematous cystitis. The presentation of ultrasound image is variable and depends on focus and amount of air. The small amount of intraluminal air results in hyperechogenicity with reverberation echoes bordering bladder wall. When it reaches a sufficient volume repeat echoes are noted down the back of anterior bladder wall; this corresponds to X-ray hydro-aeric level. As regards intraparietal air, it is characterized by hyperechoic thickening of the wall with dirty shadow cone. That impairment may be persistent, thus impeding visualization of retrovesical structures; this is one of ultrasound exam limits.

Bladder rupture is one of the dreaded complications as regards emphysematous cystitis [10]. The examination of anterior bladder wall with a linear array probe enables a better analysis of the latter. In this cohort, it helped note in the first patient an irregular aspect of the wall with presence of aeric hyperechogenicity characterized by extraparietal appearance. This gives rise to suspicion of subserous rupture confirmed by computed tomography.

Moreover, ultrasound scan helps explore the upper urinary tract in search of hydronephrosis. In some cases it helps detect subvesical obstruction.

Despite ultrasound importance in emphysematous cystitis diagnosis, computed tomography alone allows complete assessment of injuries. In this research work it enabled visualize in patient an emphysematous ureteritis and an emphysematous pyelitis suspected during ultrasound.

\section{Conclusion}

As an exploration tool, ultrasound is accessible. Therefore, it can help diagnose emphysematous cystitis especially when computed tomography is unavailable. However, computed tomography, required for complete assessment of injuries, remains the exam of reference.

\section{References}

[1] Thomas, A.A., Lane, B.R., Thomas, A.Z., Remer, E.M., Campbell, S.C. and Shoskes, D.A. (2007) Emphysematous Cystitis: A Review of 135 Cases. BJU International, 100, 17-20. http://dx.doi.org/10.1111/j.1464-410X.2007.06930.X

[2] Mnif, M.F., Kamoun, M., Kacem, F.H., Bouaziz, Z., Charfi, N., Mnif, F., et al. (2013) Complicated Urinary Tract Infections Associated with Diabetes Mellitus: Pathogenesis, Diagnosis and Management. Indian Journal of Endocrinology and Metabolism, 17, 442-445. http://dx.doi.org/10.4103/2230-8210.111637

[3] Bobba, R.K., Arsura, E.L., Sarna, P.S. and Sawh, A.K. (2004) Emphysematous Cystitis: An Unusual Disease of the Genito-Urinary System Suspected on Imaging. Annals of Clinical Microbiology and Antimicrobials, 4, 1-4.

[4] Connor, L.A.O. and Guzman, J.D.E. (2001) Emphysematous Cystitis: A Radiographic Diagnosis. American Journal of Emergency Medicine, 19, 211-213. http://dx.doi.org/10.1053/ajem.2001.23896 
[5] Eken, A. and Alma, E. (2013) Emphysematous Cystitis: The role of CT Imaging and Appropriate Treatment. Canadian Urological Association Journal, 7, 11-13. http://dx.doi.org/10.5489/cuaj.472

[6] Choong, K.K.L. (2003) Sonographic Detection of Emphysematous Cystitis. Journal of Ultrasound in Medicine, 22, 847-849.

[7] Habetz, V.J., Matthews, C.C. and Durel, R.M. (2014) Emphysematous Cystitis. Ochsner Journal, 14, 529-531.

[8] Grupper, M., Kravtsov, A. and Potasman, I. (2007) Emphysematous Cystitis: Illustrative Case Report and Review of the Literature. Medicine, 86, 47-53. http://dx.doi.org/10.1097/MD.0b013e3180307c3a

[9] Behera, V., Kumar, R.S.V., Mendonca, S., Prabhat, P., Naithani, N. and Nair, V. (2014) Emphysematous Infections of the Kidney and Urinary Tract: A Single-Center Experience. Saudi Journal of Kidney Diseases and Transplatation, 25, 823-829. http://dx.doi.org/10.4103/1319-2442.135164

[10] Viswanathan, S., Rajan, R.P., Iqbal, N., Subramani, J. and Muthu, V. (2012) Enterococcus Faecium Related Emphysematous Cystitis and Bladder Rupture. Australasian Medical Journal, 5, 581-584.

http://dx.doi.org/10.4066/AMJ.2012.1416 